\title{
Zu Theodor Lipps' Neuausgabe seiner deutschen Bearbeitung von Humes Treatise of human nature. ${ }^{\text {) }}$
}

\author{
Von Paul Wüst.
}

Die erste Auflage des I. Teiles ist 1895 erschienen. Dass nach neun Jahren schon eine neue nötig wurde, zeigt, dass die deutsche Bearbeitung von Humes Jugendwerk wirklich ein Bedürfnis war.

Mit Recht wird der Treatise of human nature $I$ in neuerer Zeit der späteren Inquiry concerning human understanding vorgezogen. Diese stand lange im Vordergrund des Interesses, weil nur sie unmittelbar für das Verständnis der von Kant an Hume geübten Kritik in Betracht kommt; denn Kant hat in der vorkritischen Zeit Humes Philosophie nicht in ihrer ersten Fassung kennen gelernt. Da Lipps auf eine historische Einleitung verzichtet, so will ich die Geschichte des Treatise in Deutschland kurz zu berichten und dabei auch die eben ausgesprochene Behauptung zu beweisen versuchen.

Hume selbst hat öfter erklärt, dass nur die Inquiry, nicht der Treatise die massgebende Fassung seiner philosophischen Prinzipien sei (vgl. Elkin, Hume: the relation of the Treatise of human nature - book I to the Inquiry concerning human understanding (1904) S. 5 f.). Er hat mit diesen Aussprüchen dazu beigetragen,

1) David Humes Traktat über die menschliche Natur (Treatise on (sic!) human nature). Ein Versuch, die Methode der Erfahrung in die Geisteswissenschaft einzuführen. In deutscher Bearbeitung mit Anmerkungen und einem Sachregister herausgegeben von Theodor Lipps.

I. Teil: Über den Verstand. Zweite, durchgesehene Auflage. Hamburg und Leipzig. Verlag von Leopold Voss. 1904. VIII u. 380 S. $8^{\circ}$.

Desgleichen:

II. Teil: Buch II: thber die Affekte (Of the passions).

BuchIII: Über Moral (Of morals).

Mit Zugrundelegung einer Übersetzung von Frau J. Bona Meyer Deutsch mit Anmerkungen und einem Index von Theodor Lipps. Ebenda 1906. VI u. 397 S. 80.

Kuntstudiou $\mathbf{X I V}$. 
den "deadborn from the press" gefallenen 'l'reatise für lüıgere Zeit selust den historischen Interesse zu entziehen, and sie sind mit die Ursache gewesen, dass noch nachdem dieses crwacht war, bis in unsere Zeit hinein das grundlegende Erstlingswerk geringer bewertet wurde als die spätere, populäre Redaktion. In den ersten Juhren nach seiner Veröffentlichung wurde der 'I'reatise kaun beachtet. Hune erzühlt in seiner Lebeusbeschreibung: $n \ldots$ in $1749 \ldots$ my former publications (all but the unfortunate 'Treatise) were beginning to be the subject of conversation ". ${ }^{1}$ ) Ganz unbeachtet blieb der Treatise indes nicht: E. Cassirer weist nenerdings nach, dass der Franzose Manpertnis schon 1746, also 6-7 Jahre nach den Erscheinen des Treatise, 2 vor dem der Inquiry, „das Humesche Problem zuerst in den Gesichtskreis der deutschen Philosoplie riickt", also auf dem Treatise fusst.") Joch haben die Philos. Essays, die bald darauf (1748) erschicnen, die Aufmerksankeit absorbiert: von der Inquiry erscheint schon nach 7 Jahren, 1755, dic erste dentsche Übersetzung, 1793 folgt eine zweite durch Tennemann. Der 'T'reatise wurde dagegen erst 1790-91 durch Jakob übersetzt. ${ }^{7}$ ) Es ist wahrscheinlich, dass die Deutschen nicht durch Maupertuis, sondern erst durch die in der deutschen Übersetzung von Beatties "Versuch ïber die Natu der Walırheit" (1772) enthaltenen Auszüge aus den 'Treatise anf dicsc erste Schrift Humes aufmerksam gemacht wurden; jenc Beattie-Übersetzung wird bis zum Erscheinen der des Treatise durch Jakob für die des Englischen nicht kundigen Deutschen die einzige Quelle für die Keuntnis des Treatise gewesen sein. Aber imner bleiben Inquiry, bezw. Philos. Essays im Vordergrund, nur diese oder ilıre Übersetzungen werden in der deutschen philosophischen Litteratur, soweit ich durch meine Stichproben feststellen kann, bis zunn Zeitpunkt der Übersetzung des T'reatise überhaupt zitiert: so bei Feder, Logik und Metaphysik (4.Aufl. 1775), S.299; bei Jaco bi, David Hume über den Glauben etc. (1787; Werke, Bd. 2 [1815], S. 128, 152 Anm. u. sonst); bei C. C. E. Schmid, Wörterbuch zum leichtern Gebrauch der Kantischen Schriften (2. Anfl. 1788), S. 100 ; in desselben Versuch einer Moralphilosophie (2. Aufl. 1792), S. 287; bei Flatt, Fragmentarische Beyträge

1) Philosophical Works ed. Green-Grose (1898) Vol. III, p. 4.

2) Das Erkenntnisproblem in der Philosophie und Wissenschaft der neueren Zeit, 2. Bd. (1907), S. 334. 687 f. (Aum. 27).

3) Vyl. auch Lipps I, V. 
zur Bestimmung und Deduktion deş Begriffs und Grundsatzes der Caussalität (1788), S. 5 u. sonst.

Von Bedeutung ist es, dass weder Schmid noch Flatt, deren Schriften sich ausdrücklich auf die Kants beziehen, eine Anspielung darauf machen, dass dieser den Treatise gekannt habe; auch die Übersetzung von Beatties Werk wird nicht erwähnt. Kant selbst zitiert Hume direkt nur einmal, und zwar nach der deutschen Übersetzung der Essays (Prolegomena ed. Kehrbach, S. 32 Anm. Vgl. A. Riehl, Der philosophische Kritizismus I ', S. 69 f.). Beatties Buch in der Übersetzung von 1772 hat bei Kant offenbar kein Interesse für den Treatise erweckt, noch weniger lässt sich bei ihm ein Einfluss der darin ausgezogenen Stellen von Humes Jugendwerk nachweisen - so mit B. Erdmann, Reflexionen Kants zur kritischen Philosophie Bd. 2, S. LX gegen Vaihinger, Kommentar zu Kants Kr. d. r. V., I, S. 347 und L. Ducros, Quando et quomodo Kantium Humius e dogmatico somno excitaverit (1883), S. 30 f. ${ }^{1}$ )

Es scheint also gewiss, dass Kant den Treatise nicht vor jener ersten deutschen Übersetzung durch Jakob, die nach den Kritiken und den Prolegomena erschien, kennen lernte: soviel wir wissen, war er ja auch des Englischen nicht kundig. Das , erscheint nach den Ausführungen Erdmanns (Archiv f. Geschichte der Philosophie Bd. I (1888), S. 62 ff., bes. S. 65 f.) sicher. Die von Vaihinger, KSt. Bd. 5 (1900), S. 114 angefïhrte Briefstelle, wonach Kant „The Gentleman's Magazine“ besessen habe und also des Englischen mächtig gewesen sein müsse, darf offenbar zu so weitgehenden Schlüssen nicht berechtigen; ihnen steht das von Erdmann a. a. 0 . angeführte Zeugnis Jachmanns gegenüber. Kants Freund Green konnte ihm wohl einiges aus dem „Maga-

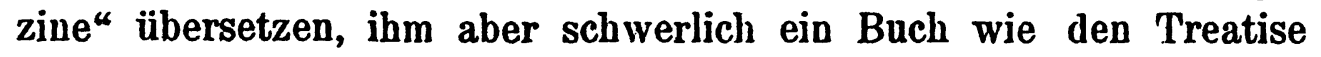
auslegen. So wagt denn auch $G$ roos in seinem kleinen Aufsatz KSt. Bd. 5, S. 177 ff. nur die Möglichkeit einer Bejahung seiner Hrage „Hat Kant Humes Treatise gelesen?" zu behaupten. Dass am Schlusse des 1. Buches des Treatise und in den Prolegomena (a. a. O. S. 37) das gleiche Bild vom Ozean gebraucht wird, hätte Groos nicht als Stütze für seine Vermutung anfülıen

1) Ducros sagt a. a. O., Vaihinger missverstehend, Humes „Treatrise" (so überall!) sei 1772 von Beattie ins Deutsche übersetrt worden (!). D. hat das betr. Buch offenbar nie sellsst in der Hand gehabt. 
dürfen: Vaihingers Kommentar (a. a. O. S. 39 f., bes. auch S. 40, Anm. 2) konnte ihm zeigen, dass solche rein stilistischen Ähnlichkeiten gar nichts beweisen; jenes Bild wird von Kant z. B. schon 1763 im „Beweisgrund ${ }^{\alpha}$ gebraucht (vgl. Ausgabe Rosenkranz I, S. 164), also zu einer Zeit, wo Kant von Hume jedenfalls noch gar nichts, am allerwenigsten aber den Treatise gelesen hatte; und späterhin bringt Kant das Bild so überaus häufig, wie nur ein selbstgeschaffenes, nicht aber ein entlehntes verwendet wird.

All diese äusserlichen Beweismomente würden bereits genügen, um die Behauptung, Kant habe Humes Lehren auch in der Fassung des Treatise gekannt, abzulehnen. Dazu treten schwerer wiegende innere Gründe, die schon oft erörtert wurden, und auf die ich deshalb hier nur kurz hinweise: das wichtigste Moment liefert eine Bemerkung Kants in der Kr. d. r. V. (Ausg. Kehrbach, S. 584 f.): „Die skeptischen Verirrungen aber dieses sonst äusserst scharfsinnigen Mannes entsprangen vornehmlich aus einem Mangel, den er doch mit allen Dogmatikern gemein hatte, nämlich, dass er nicht alle Arten der Synthesis des Verstandes a priori systematisch übersah. Denn da würde er, ohne der übrigen hier Erwähnung zu tun, z. B. den Grundsatz der Beharrlichkeit als einen solchen gefunden haben, der eben sowohl, als der der Causalität, die Erfahrung antizipiert ..." Die Bemerkung Kants trifft nur die Inquiry, da Hume im 'Treatise dem von Kant vermissten Problem lange Erörterungen gönnt, deren kritisch-negativer Wert m. W. zuerst von P. Natorp, Einleitung in die Psychologie nach kritischer Methode (1888), S. 58 betont worden ist.

Kants Kritik geht also, rein geschichtlich genommen, nicht anf den ganzen Hume. Untersuchungen, wie die von Kühne, ${ }^{1}$ ) Ritter ${ }^{2}$ ) und Schultze ${ }^{\mathbf{3}}$ ) haben sich daher lediglich an die Inquiry gehalten. Wenn nun auch die treffliche Arbeit von Elkin (s. 0.), durch welche alle früheren das Verhältnis der beiden erkenntnistheoretischen. Schriften Humes behandelnden Abhandlungen entbehrlich gemacht worden sind,4) ergeben hat, dass die spätere theorie.

1) Über das Verhältnis der Humeschen und Kantischen Erkenntnis-

7) Kant und Hume, Diss. 1878.

3) Hume und Kant über den Kausalbegriff, Diss. 1870.

9) Vgl. meine Anzeige im Literarischen Zentralblatt 1905, Sp. 1773 ff. 
Schrift in Methode und Tendenz wie im Ergebnis keine wesentliche Abweichung vom Treatise aufweist, so zeigt uns doch gerade Elkins Darstellung die Wichtigkeit dieser ersten Schrift. Denn einmal ist rein sachlich alles nicht in die Inquiry Aufgenommene von Interesse; dies gilt besonders für den „wertvollen kritischen Ansatz“ in Humes Erörterung der Substanzialität (Natorp); dann auch kann der Treatise allein uns Aufschluss darüber geben, ob Kant Recht hat mit seiner Behauptung, Hume „würde ... dem a priori sich erweiternden Verstande und der reinen Vernunft bestimmte Grenzen haben vorzeichnen können“ und also vor „skeptischen Verirrungen “ bewahrt geblieben sein (Kr. d. r. V. a. a. O.), wenn er den Grundsatz der Beharrlichkeit untersucht hätte. Überhaupt giebt uns der Treatise ein genaueres Bild von Humes Philosophie als die tendenziös gekürzte spätere Schrift, indem sie zugleich das Verständnis der letzteren vertieft. Hier haben wir fast nur Ergebnisse, nur das Wenige, was durch Neuheit eine Wirkung auf den Leser verheisst - dort die frische Fülle der Einzeluntersuchungen und Ableitungen. Nur aus diesen heraus lassen sich jene voll begreifen; und so hebt uns das Studium des Treatise aus der oben gekennzeichneten Sphäre engerer geschichtlicher Betrachtung Humes und seines Verhältnisses zu Kant auf eine höhere Stufe, wo die geschichtliche Vergleichung sachlich vertieft wird; wo der ganze Hume an Kant gemessen werden kann.

Der erste, welcher energisch auf die Notwendigkeit einer Ergänzung der Inquiry durch den Treatise, ähnlich wie auf das Verhältnis der beiden ersten Auflagen der Kr.d. r. V. zueinander hinwies, war Jacobi. Er schrieb: „Überhaupt wird nicht genug erkannt, welchen Vorteil es gewährt, die Systeme grosser Denker in den frühesten Darstellungen derselben zu studieren. So erzählte mir Hamann von ... Kraus, dass dieser nie hatte aufhören können, ihm dafür zu danken, dass er ihn mit Humes erstem philosophischen Werke: Treatise of human nature, 1739 , bekannt gemacht, weil ihm hier erst das wahre Licht über die späteren Essays aufgegangen wäre. ${ }^{1}$ ) Seit Jacobi ist man der Bedeutung des Treatise in steigendem Masse gerecht geworden: nur diese Schrift und die späteren geben uns den ganzen Hume. Nach

1) Jacobi a. a. O. S. 291. Vgl. Pfleiderer, Empirismus und Skepsis in David Humes Philosophie (1874), S. 100 u. Anm. 
Hedvall ${ }^{1}$ ) ist "die immer mehr zunehmende Neigung, den Treatise an dic erste Stelle zu setzen ... in dem Masse gestiegen, als Hume mehr und mehr aktuell') geworden".

Die erste Auflage von Band I der Lippsschen Bearbeitung hat nicht geringe Verdienste daran, dass Hedvalls Bemerkung besonders auf die Zeit seit 1895, dem Jahr eben der ersten Auflage, verstärkte Anwendung finden darf. Hierbei bedeutet der Ausdruck "Aktuellwerden" nicht bloss ein wachsendes Bedürfnis nach einem in dem oben skizzierten Sinne vertieften Studium Humes, sondern auch das häufigere Auftreten der Tendenz, Hume gegenüber seinem historischen Gegner Kant höher za bewerten. Lipps schrieb 1895 in seiner Vorrede: „Welcher der beiden Philosophen das Problem der Erkenntnis schärfer und tiefer gefasst, wer von ihnen als der grössere Entdecker auf diesem Gebiete zu gelten habe, von wem wir auch heute noch das meiste lernen können, das mag hier dahingestellt bleiben; obgleich ich meine, vorhersagen $\mathrm{zu}$ können, dass man in 7ukunft hierüber anders urteilen wird, als man jetzt noch, wohl gar mit dem Anspruch der Selbstverständlichkeit, zu urteilen gewohnt ist." Es steht ausser allem Zweifel, dass Lipps damals der Überzeugung war, die Zukunft gehöre weniger der Kritik der reinen Vernunft, „dem einzigen der Geschichte der neueren Philosophie angehörigen Werke gleichartigen Inhalts, das mit ihm (scil. dem Treatise I) verglichen werden kann", als vielmehr Humes Treatise.

Die Erwartung Lipps' hat sich nicht erfüllt. Zwar scheint es, als ob er Recht behalten sollte mit den angeführten prophetischen Worten, wenn die neueste Monographie von 0. Söhring, „David Humes ,Skeptizismus', ein Weg zur Philosophie“, die ich an anderem Orte ausführlich erörtert habe, ${ }^{8}$ ) Hume auf Kosten Kants verherrlicht; doch scheint es nur so. Denn die Beweis-

1) Humes Erkenntnistheorie kritisch dargestellt. Eine Untersuchung über empiristische Prinzipien I (Uppsala Universitets Arsskrift 1906. Filosofi, Språkvetenskap och historiska Vetenskaper 1), S. 88; vgl. meine Anzeige im Liter. Zentralbl. 1908, No. 5, Sp. 156 f.

8) Von mir gesperrt.

3) In einem der Philosophischen Wochenschrift, herausgeg. von H. Renner, überlassenen Aufsatz „Beiträge zur Hume-Interpretation“, dessen Manuskript indes nach dem lautlosen Eingehen der $\mathrm{Ph}$. W. vom ehem. Herausgeber trotz Inanspruchnahme der Behörde nicht mehr zu erlangen ist. 
führung Söhrings entbehrt völlig der Unbefangenheit. Ferner lässt sich nicht verkennen, dass die neueste Litteratur über Hume, die nach einer unbefangenen Würdigung trachtet, zwar die Verdienste Humes in der Teschichte der Philosophie würdigt und hervorhebt, sich jedoch durchweg von einer überschwänglichen Einschätzung seiner absoluten Leistung bes. Kant gegenüber freihält: die Arbeiten von Elkin, R. Hönigswald, ${ }^{1}$ ) und das Humekapitel bei Cassirer,2) um nur die wichtigsten zu nennen, mögen als Beispiele dafür gelten, dass wir einer objektiven Bewertung von Humes Erkenntnistheorie immer näher kommen. Und, last not least: Lipps selbst ist in den neun Jahren, die zwischen der ersten und zweiten Auflage von Band I seiner Übersetzung liegen, zu einem andern Werturteil gekommen: seine eigene philosophische Entwickelung hat ihn von Hume weiter ab und näher zu Kant hingeführt. Der Kenner der Lippsschen Philosophie konnte diese Wendung bereits seit geraumer Zeit verfolgen: sie tritt in der zweiten Auflage von Bd. I seiner Übersetzung von Humes Hauptwerk unzweideutig hervor, und wenn in Zukunft das Übergewicht Kants über diesen allgemeiner wird anerkannt werden, somit die alte Prophezeiung von 1895 zu Schanden kommt, so wird Lipps für Hume gegen Kant keine Lanze mehr brechen.

Die Neuauflage von $\dot{I}$ ist nun schon seit vier Jahren im Handel, doch bin ich ihr in der Humeliteratur der letzten Jahre noch nicht begegnet; es wird immer noch die alte zitiert, und das ist schade. Damit soll die Trefflichkeit des alten Buches nicht herabgesetzt werden, dessen Text für die Neuauflage nur einer stilistischen Feilung bedurfte. Aber indem man nach Erscheinen der 2. Auflage noch immer die von 1895 nennt, wird Lipps für den längst überwundenen Standpunkt, den er Hume gegenüber in der Vorrede und den Anmerkungen der 1. Auflage einnahm, noch verantwortlich gemacht. So geschieht Lipps Unrecht, wenn z. B. neuerdings (Ende 1907) Cassirer (a. a. O. S. 677) aus der 1. Auflage, wo Lipps auch schon kein kritikloser Bewunderer Humes war, eine die Humesche Custom-

1) Über đie Lehre Humes von der Realität der Aussendinge (1904); vgl. meine Anzeige im Liter. Zentralbl. 1905, No. 47, Sp. 1573 f.

2) Das Erkenntnisproblem Bd. 2, S. 244-283 u. sonst. 
theorie kritisierende Anmerkung Lipps' zitiert und dann hinzufügt: "So zutreffend dieses Urteil im allgemeinen ist, so wenig versteht man, wie Lipps hiernach das Werturteil, das er in der Vorrede über Humes T'reatise und sein Verhältnis zur Kritik der reinen Vernunft gefällt hatte, noch aufrecht zu erbalten vermag." Denn in der Neuauflage hält ja Lipps dieses Werturteil nicht mehr aufrecht, ${ }^{1}$ ) und es ist deshalb an der Zeit, durch eine ausführliche Anzeige auf die Neubearbeitung, die hinfort als die massgebende $\mathrm{zu}$ gelten hat, und zwar vor allem auf die in den Anmerkungen vorgenommenen Änderungen aufmerksan zu machen. Auch wäre es im äusseren Interesse der guten Sache wahrlich schade, wenn diese tiefgehende Revision des eigenen Standpunktes, die bei einem Philosophen von dem Rufe Lipps' eine allgemeinere, höhere Bedeutung gewinnt, länger im Dunkel bliebe. Denn nur sehr selten überschreitet nach ehrlichem, langem Ringen mit sich selbst ein Denker jene Kluft, die zwischen den philosophischen Überzeugungen besteht, solange es ein I)enken über die Grundlagen unserer Erkenntnis giebt. Dass es hier geschehen ist, bedeutet, ich darf wohl sagen, einen Triumph des still aber gewaltig wirkenden Genius' Kants. Dies Gefühl der reinen Freude über den Erfolg der Sache ist es wohl wert, dass ihm in dieser Zeitschrift, die der Förderung der Kantischen Sache dient, „den Humeepigonen unserer Tage“. (Hedvall) gegenüber laut und deutlich Ausdruck gegeben wird.

Graf Hoensbroech schrieb kürzlich einmal: das alte, starre und lebensfeindliche Wort: " "Was ich geschrieben habe, das habe ich geschrieben", ist nirgendwo mehr verbreitet, als in der Gelehrtenwelt". Die Änderungen, die Lipps an seinem Buche vorgenommen hat, beweisen, dass ihm ein solch unfreies Festhalten an dem einmal öffentlich Geäusserten fernliegt. Aber er hat, so scheint es, nicht laut darauf hinweisen wollen, dass die neue Auflage auf einem wesentlich anderen Standpunkt steht als die alte. Diese Tatsache wird durch den Zusatz "durchgesehene Auflage" nicht so deutlich zum Ausdruck gebracht, wie es ihrer Bedeutung entsprochen hätte. Ferner ist die Überschrift S. V : „Aus dem Vorwort zur ersten Auflage der

1) Vgl. auch die Änderungen und Zusătze der 2. Auflage bei der betr. Anmerkung (282) unten S. 262. 
Übersetzung " und die Beibehaltung des Datums 1894 für den, der die erste Auflage nicht kennt und sie nicht mit der zweiten verglichen hat, irreführend: man glaubt, die neue Vorrede enthalte die wesentlichen Erörterungen der alten unter Weglassung der minder wichtigen, unwesentlichen. Die neue Vorrede ist aber innerlich, ich möchte sagen der Gesinnung nach, eine völlig andere; sie würdigt nur noch das geschichtliche Verdienst Humes und enthält keine absolute Bewertung mehr; die Kürzungen ${ }^{1}$ ) treffen nämlich gerade diejenigen Stellen, die der alten Vorrede ihr charakterisches Gepräge, das einer Werbeschrift, eines Aufrufes zum Studium der Humeschen Philosophie gegeben hatten. Warum hat es Lipps verschmäht, die Abwendung von Hume, welche die Neuauflage offenbar darstellt, hier in der Vorrede rückhaltlos zu berichten, so offen, wie sie in den Änderungen vieler Anmerkungen zu Tage tritt?

Gestrichen sind in der 2. Auflage z. B. folgende Stellen aus der Vorrede der ersten:

1. Diejenige, welche das von Cassirer a. a. 0 . beanstandete, oben schon zitierte Werturteil über Humes Treatise und sein Verhältnis zu Kants Kr. d. r. V. enthält.

2. „Welche Stellung und Bedeutung man auch sonst der „Abhandlung über die menschliche Natur" anweisen mag, in jedem Falle ist sie durch nichts ersetzbar für denjenigen, dem daran liegt, an der Hand eines der Geschichte angehörigen Philosophen in philosophische Probleme sich einführen zu lassen. Hume ist der Meister in der Kunst der psychologischen Analyse, durch die allein das Erkenntnisproblem zu lösen ist, $\left.{ }^{2}\right)$ und die für alle sonstige philosophische Arbeit die Voraussetzung bildet. Dazu kommt, dass Hume zu den klarsten Schriftstellern gehört. Hume ist klar auch in seinen Irrtümern. Er ist zugleich der lebendige Beweis dafür, dass wahre Tiefe mit Klarheit und Einfachheit nicht unverträglich ist. Hume führt überall in die Tiefe; aber er meidet den blossen Schein der Tiefe. Diese wissenschaftliche Wahrhaftigkeit und die daraus fliessende Bescheidenheit des Wissens, das sind nicht die letzten unter den Eigenschaften,

1) In der Neuauflage sind auch die weggelassenen Stellen nirgendwo durch ... markiert.

2) Von mir gesperrt. 
die die ${ }_{n}$ Abhandlung über die menschliche Natur ${ }^{\text {" geeignet }}$ machen, in die Philosophie einzuführen".

3. In dem Satze: "Die Kritik Humes, die keine so leichte Sache ist, wie manche anzunehmen scheinen, ${ }^{2}$ ) blieb ausgeschlossen " die gesperrten Worte.

Ich habe schon oben bemerkt, dass Lipps bereits in der ersten Auflage gelegentliche Kritik an Hume keineswegs ausschloss. Wie schon die zuletzt notierte Streichung erwarten lässt, muss Lipps auf seinem jetzigen Standpunkte eine solche noch weniger unmöglich erscheinen. In der Tat lassen es die Anmerkungen der 2. Auflage an bisweilen recht scharfer Kritik nicht fehlen, und ich verstehe daher nicht völlig, warum Lipps den unter 3. zitierten Satz nicht ganz strich. Aus den Anmerkungen geht ferner hervor, dass alles unter 1. 2. Angeführte nicht mehr zu Lipps' heutiger Auffassung passt, durch die Weglassung in der 2. Auflage wird es aber nicht ausdrücklich als beseitigt und ungültig bezeichnet. -

Im folgenden werde ich an den Stellen, wo die Veränderung in Lipps' Bewertung der erkenntnistheoretischen Leistung Humes besonders deutlich erkennbar wird, den Wortlaut der ersten und der zweiten Auflage einander gegenüberstellen.

1. Auflage.

Anmerkung 4.

... Hieraus ${ }^{1}$ ) erklären sich die meisten Sonderbarkeiten H.umes, ${ }^{2}$ ) z. B. bei Besprechung der Mathematik

$$
\text { ( }
$$

Dies schliesst jedoch nicht aus, dass gerade bei Hume für das Verständnis des begrifflichen Denkens die bedeutungsvollsten²) Ansätze vorliegen.
2. Auflage.

Anmerkung 4.

... Darin liegt der grosse Mangel Humes, der ihn erkenntnistheoretisch scheitern lässt. Er liegt in diesem „Psychologismus", den völlig zu überwinden jetzt die dringendste Aufgabe der. Lehre ${ }^{\circ}$ vom Verstand $\theta^{\prime \prime}$ ist. $\left.{ }^{8}\right)$ Dies schliesst doch nicht aus, dass gerade bei Hume für das Verständnis des begrifflichen Denkens bedeutungsvolle?) Ansätze vorliegen.

1) d. i. aus der „Verkennung des eigenartigen Wesens des begrifflichen Denkens", aus der Vermischung des Denkens, „das auf Gegenstănde geht . . . mit dem Vorstellen, d. h. dem Haben von Inhalten oder Bildern ...."

2) Von mir gesperrt. 


\section{Auflage.}

Anmerkung 8.

... Diese $Z$ weideutigkeit ${ }^{1}$ ) im Begriff der perception (scil.: perception $=$ Akt, Inhalt und Gegenstand) ist wohl zu beachten, sie stört aber die Klarheit der Darstellung Humes nicht.1)

\section{Anmerkung 29.}

... Es sei darauf aufmerksam gemacht, dass Hume hier ... nicht von der Identität redet, die ein nichtssagender Satz der Identitat proklamiert, d. h. nicht von der Identitat, 1 ) die einem jeden Objekt in einem jeden Moment mit sich selbst eignet.

Anmerkung 76. (nidea bei Hume auch "Vorstellung" im Sinne von Vorstellungsinhalt.)

Anmerkung 84.

... Unter der Existenz versteht Hume hier lediglich das Dasein der Eindrücke und Vorstellungen selbst.1)
Anmerkung 8.

2. Auflage, ... Vor allem der Begriff der perception wird vermöge dieser $\mathrm{Nicht}$ unterscheidung unheilvoll. Alles ist für Hume schliess. lich perception $\left.{ }^{1}\right)$...

Anmerkung 29.

Wie in der 1. Auflage, doch ist das Gesperrte gestrichen.

Anmerkung 76.

Desgleichen, doch mit dem Zusatz: Die ganze Beweisführung Humes in diesem Kapitel über Raum und Zeit beruht darauf, dass er von diesem Inhalt den darin gedachten Gegenstand, der einer vollig anderen, nämlich der logischen oder Denkgesetzmăssigkeit unterliegt, ${ }^{1}$ ) nicht unterscheidet.

\section{Anmerkung 84.}

Desgleichen, doch mit dem Zusatz: und Vorstellungen bezw. ihrer Inhalte; er unterscheidet aber davon freilich die Existenz der darin gedachten Gegenstände nicht.1)

Text S. 91. ${ }_{n}$ Es kann sich . . . kein Gegenstand dem Bewusstsein darstellen, der einem Gegenstand bezüglich seiner Existenz gleich und von anderen in derselben Hinsicht verschieden wăre, weil eben jeder Gegenstand, der. sich dem Bewusstsein darstellt, notwendigerweise 90) existieren muss."

1) Vón mir gesperrt. 
Anmerkung 90.

1. Anflage.

Wenn auch nur im Bewrosstsein.

Anmerkung 149.

... survey ... ist der Akt der inneren Erfahrung von etwas ...

Anmerkung 156.

... die ... Ausdrücke (solidity, firmness, steadiness) zeigen besonders deutlich, was Hume will und nicht will... Phantasiegebilde können höchst lebendig oder lebhaft sein; damit ist doch an sich noch nichts gegeben von dem, was Hume bei der Erörterung des Glaubens als Lebendigkeit der Vorstellungen bezeichnet. Was er meint, ist kurz gesagt, der Charakter der "Objektivitat", die Art gewisser Bewusstseinsobjekte, sich als etwas von uns Unabhängiges zu geberden, ${ }^{1}$ nicht unserer Willkür sich zu fügen, sondern uns in unserem Vorstellen zu nötigen, sodass wir ihnen gegenüber ein unmittelbares Bewusstsein unserer eigenen Passivität haben. Das Bewusstsein jener Objektivität oder dieser Passivität ist das feeling, 1 ) von dem Hume hier redet, es ist die Anerkennung, der Glaube."

1) Von mir gesperrt.
Anmerkung 90.

2. Auflage.

Fr existiert notwendig im Bewusstsein; aber von dieser subjektiven Existenz unterscheidet Hume die objektive nicht; die aussere Existenz fliesst ihm zusammen mit der Existenz der ausseren, d. h. der sinnlichen Perzeptionen.

Anmerkung 149.

Desgleichen, doch mit dem Zussatz: Aber auch dies fliessi für Hume mit dem Vorstellen oder Perzipieren zusammen. Er weiss auch hier nichts von dem Besonderen des Denkens.1)

Anmerkung 156.

... die

$$
\begin{gathered}
1 \\
\text { desgleichen } \\
(
\end{gathered}
$$

meint, aber nicht unzweideutig sagt, oder, was er sucht, aber nicht findet, ist das mit jedem sonstigen Datum unseres Bewusstseins Unvergleichbare:1) das "empirische Objektivitätsbewusstsein ", das Bewusstsein "der Forderung" oder des "Rechtes", nicht der Vorstellung, noch auch des Inhaltes derselben, sondern des darin ,gemeinten Gegenstandes", gedacht zu werden, das Bewusstsein des objektiven, d. h. dem Gegenstande eigenen Dasein, kurz der Geltung oder "Gültigkeit" des Denkaktes. Hierin besteht in Wahrheit das feeling, $\left.{ }^{1}\right)$ von dem Hume hier redet. 

1. Auflage.
2. Auflage.

Text S. 148 (Mitte). ... der Geist . . . fasst . . . diese Vorstellungen in ein neues System zusammen, das er . . . mit dem Namen „Wirklichkeit ${ }^{4}$ beehrt.

\section{Hierzu:}

Anmerkung 176.

Es verdient Beachtung, dass hier deutlich die [objektive] Notwendigkeit, die Art der Objekte unserer Vorstellungswillkür entrückt zu sein, als der wesentliche Inhalt des Wirklichkeitsbewusstseins erscheint.
Anmerkung 176

gestrichen.

Text S. 148 (oben). . . innere Perzeption ...

\section{Hierzu:}

keine Anmerkung.

\section{Anmerkung 191.}

. . . er bezeichnet, was wir spontane Tätigkeit des Denkens nennen würden, als Tätigkeit der Einbildungskraft. Damit degradiert er nicht diese Tätigkeit; als ob wissenschaftliche Terminologie degradieren könnte; sondern er wehrt die übliche Degradierung des Begriffs der Einbildungskraft ab. Zugleich lässt er deutlich werden, dass unsere gewöhnlichsten „Einbildungen" doch auch im letzten Grunde denselben Faktoren ihr Dasein verdanken, wie unsere sichersten Erfahrungserkenntnisse, 1) dass nicht etwa für die letzteren ein besonderes mystisches Vermögen erst noch erfunden $z u$ werden braucht. ${ }^{1}$ Mit einem Worte: Hume erklärt, statt sich an Worte zu hăngen.')
Anmerkung 176.

D. h. als impression of reflexion. Richtiger wäre: Perzeption eines Inneren.

Anmerkang 191.

...er

$$
\left.\right|_{\text {desgleichen: }}
$$

mit kleiner stilistischer Ändernng.

wehrt eine übliche Einengung des Begriffs der Einbildungskraft ab. Zugleich liegt darin freilich die Meinuug, dass unsere gewöhnlichsten "Einbildungen" im letzten Grunde denselben Faktoren ihr Dasein verdanken, wie unsere sichersten Erfahrungserkenntnisse.1) Diese Meinung findet ihre Erklärung in dem Umstande, - an dem schliesslich diese ganze Abhandlung überden Verstand scheitert, - dass Hume das spezifische Wesen der Denktătigkeit, des Logischen in uns, kurz, des "Verstandes" nicht sieht.1)

1) Von mir gesperrt. 
Alimerkung 282.

\section{Auflage.}

Hiermit (scil. der Lelire, dass die Geuolmheil der Beobachtning daucrndes. Kohïrenz der Walurnelumungen die $L_{r}$ sache des Glaubens an die dausende Existenz der Gegenstände sei) ergänzt oder vervollståndigt Hume . . . sein Prinzip der Gewohnheit. Zugleich gewinnt das Prinzip damit einen neuen und erst eigentlich 10gischen Charakter; es wird zu einer Art von allgemeinem Prinzip der 'Trägheit, Konstanz, Konsequenz, kurz Gesetzmässigkeit des denkenden Geistes. ${ }^{1}$ ) Ein Schritt weiter in dieser Richtung, und das Gewolnnheitsprinzip als solches, die vermeintliche logische Bedeutung des Gewohnheitsmässigen oder Angewölınten schwindet und das reine Gesetz der Gesetzmässigkeit des Geistes, ${ }^{1}$ ) die apriorische Tatsache, dass überhaupt das menschliche Denken gesetzmässig ist, die Tatsache also, auf der in Walkrheit alles Schliessen beruht und aus der insbesondere das Kausalgesetz ohne Weiteres sich ergiebt, bleibt übrig. Hume hat diesen Schritt nicht get:an.1)
Anmerkung 282.

\section{Auflage.}

Hiermit ... .

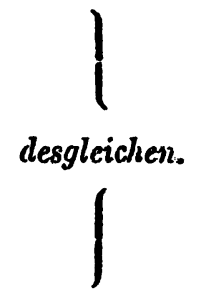

neuen Charakter, derart, dass es fast wie ein logisches oder Erkenntnisprinzip sich ausnimm $t^{1}{ }^{1}$ ) es wird zu einer Art von allgemeinem Prinzip der Konstanz, Konsequenz, kurz Gesetzmässigkeit des "Geistes".1) Ein Schritt weiter in dieser Richtung, und das Gewolnheitsprinzip als solches, die vermeintliche logisclie Bedentung des Gewohnheitsmässigen oder Angewöhnten musste fallen und die wirkliche Gesetzmässigkeit des Geistes im engeren und spezifischen Sinne dieses Wortes, ${ }^{1}$ ) die apriorische Gesetzmässigkeit des Denkens, die Eines ist mit der Gesetzmassigkeit der gedachten Gegenstände, ${ }^{1}$ ) und in der auch das Kausalgesetz beschlossen liegt, wurde von Hume gefunden. Hume hat diesen Schritt nicht getan. Kein Wunder: Er hätte zunăchst das eigenartige, und von jedem blossen Perzipieren, oder Haben von Bewusstseinsinhalten, toto coelo verschiedene Wesen des Denkens,1) oder was dasselbe sagt, er hätte den. Begriff des dem Inhalte transscendenten ,Gegenstandes" finden unussen. Und dies hatte ein Umdenken sei. nes ganzen Buches „über den Verstand" bedeutet.1)

1) Voll mir gesperrt. 


\section{Auflage.}

Anmerknng 302.

Hume: internal perceptions; nicht innere im Gegensat\% zu iusseren Per\%eptionen; 1 ) sondern Perzeptionen als innere Vorgänge, also iiberhaupt: innere Vorgänge. Denn alle ,inneren" Vorgänge fallen für Hume unter diesen Begriff.

Anmerkung 308.

Fs isthier, wie in so manchen Fillen, leicht auf Humes Kosten geistreich zu sein, wenn man nicht beachtet, worum es sich handelt.1; Hier wird zumächst vielleicht mit unütiger Unständlichkeit eingeschärft, dass es wie Vorstellungen, so auch Findrïcke giebt, denen das Merkmal der Ausdelinung zukommt. Die Vorstellung des Ausgedehnten, die wir ja jedenfalls haben, ist nur möglich als Nachbild eines ihr Gleichartigen, also eines Ausgedehnten, das vorher als Findruck dem Bewusstsein gegenwärtig war. Daher versteht Hume, wie iiberall, unter den Eindrïcken und Vorstellungen, er versteht ebenso nachher unter den Perzeptionen, die Findrïcke und Vorstellungen umfassen, nicht die Akte des Wahrnehmens und Vorstellens, die ja auch unserer unmittelbaren Erfalırung gar nicht gegeben sind, sonderu lediglich als Bewusstseinsoljekte, beispielsweise den jetzt von mir walhrgenommenen oder vorgestellten Tisch. Hr wendet sich gegren diejenigen, die meinen, es habe Sinn zu sagen, diese Bewusstseinsobjekte seien [räumlich] ${ }_{n}$ in $^{\text {" der Seele oder }}$ der vorstellenden „Substanz ${ }^{\mu}$. Er spielt im folgenden insbesondere mit denjenigen, die die ausgedelınten
2. Auflage.

Anmerkung 302.

Hume: internal perceptions; innere oder nach innen gerichtete Perzeptionen. Wiederum werden hier die Perzeptionen nicht unterschieden von dem, was darin perzipiert wird, ihrem Gegenstande.1)

\section{Anmerkung 308.}

Die Konsequenz der grundsätzlichen Verwechselung von Akt der Perzeption und Perzipierte ${ }^{1}$ ) tritt hier besonders dentlich жutage. - Da für Hume der Gegenstand mit der Perzeption zusammenfällt, so ist natürlich für ihn die Vorstellung des Gegenstandes mit der „Vorstellung der Perzeption" gleichbedeutend.

1) Von mir gesperrt. 


\section{Auflage.}

Bewusstseinsobjekte oiner einfachen oder unausgedehnten Seele raumlich einverleiben (incorporate) wollen, indem er fragt, wie eigentlich sie sich ein solches raumliches $\mathrm{Zu}$ sammen vorstellen wollen, da man. sich doch sonst bei Worten etwas vorzustellen pflegt. Kurz er sucht zu zeigen, dass diese ganze Verräumlichung der Beziehung der Perzeptionen zur Seele so widersinuig ist, wie es tatsăchlich ist.

\section{Anmerkung 310.}

Wenn wir von dem Inhalt oder. Objekt des Bewusstseins ${ }^{1}$ ) (perception) den (wirklichen) Gegenstand unterscheiden, so ist die ,Vorstellung " des letzteren in Wahrheit nichts, als der gänzlich inhaltleere Gedanke eines Etwas, das zu dem Bewusstseinserlebnis in irgend welcher Beziehung steht, oder sie ist, wie jede inhaltlich irgendwie bestimmte Vorstellung, Nachbild einer Wahrnehmung. Mag der (wirkliche) Gegenstand an sich sein, was oder wie er will, für unser Bewusstsein (für die Wahrnehmung oder Vorstellung, 1) ist er doch immer nur ein Inhalt der Wahrnehmung ${ }^{1}$ ) oder der Wahrgenommenes reproduzierenden Vorstellung.

\section{Auflage.}

Anmerkung 310.

Wenn wir von dem Bewusstseinserlebnis ${ }^{1}$ ) (perception) den

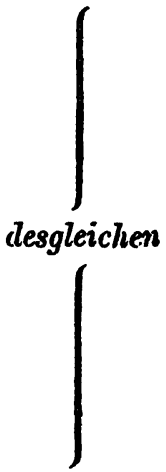

will, für unser Bewusstsein ist er doch immer nur ein Wahrnehmungsoder ein die Wahrnehmung reproduzierendes Vorstellungserlebnis. - Dies ist ebenso unzutreffend, wie es einfach klingt. "Bewusstsein" ist nicht nur Wahrnehmung oder VorstelIung: sondern auch Denkén. ${ }^{1}$ )

Text S. 327. ... ich kann wagen, von allen ... Menschen zu be= haupten, dass sie nichts sind als ein Bündel oder Zusammen 316) verschiedener Perzeptionen ... Der Geist ist eine Art Theater, auf dem verschiedene Perzeptionen nacheinander auftreten ...

1) Von mir gesperrt. 
1. Auflage.

Anmerkung 316.

Hume: bundle or collection.
2. Auflage.

Anmerkung 316.

Hume: bundle or collection. Hume übersieht, dass auch ein "Bündel“ oder "Zusammen" mehr ist als eine Menge, dass es ein Bindendes oder Zusammenfassendes in sich schliesst.1) Das gleiche gilt vom "Theater".

Die Änderungen der 2. Auflage zeichnen sich zunächst durch ihre negative Seite ans: diese bringt zum Ausdruck, dass Lipps jetzt dem Grundgedanken des Treatise, der Begründung der Erkenntnis in den einzelnen psychischen Akten, kurz, dem „Psychologismus" Humes durchaus skeptisch gegenüber steht. Während ihm in der ersten Auflage noch die psychologische Analyse als dasjenige Mittel galt, durch welches „allein das Erkenntnisproblem zu lösen" sei (Vorrede), spricht es die Anmerkung 4 der 2. Auflage klar aus, dass" es "die dringendste Aufgabe der Lehre „vom Verstande“ ist,“ den Psychologisnus „völlig zu überwinden“. Dieses negative Moment der neuen Auflage, das aber schon nicht mehr bloss negativ ist, sondern bereits auf den positiven Fortschritt der Lippschen Auffassung der Verstandeslehre vorbereitet, kommt ausser durch die Weglassungen der oben angeführten Partien der Vorrede besonders noch bei den Veränderungen der Aumerkungen 8, 149, 191, 282 zum Ausdruck. Zugleich macht die Neubearbeitung der Anmerkungen 4, 149, 191, 282 mit der von 156, 310, 316 deutlich, was Lipps an die Stelle des „Psychologismus" gesetzt wissen möchte: die Erkenntnis der Eigenart des begrifflichen Denkens, welche Hume nicht aufgegangen war, da sich das Denken für ihn aus der Wahrnehmung ablöste. Die übrigen Veränderungen zeigen, dass Lipps die Hauptursache von Humes Verkennung der Eigenart des Denkens als einer von Wahrnehmen und Vorstellen wesentlich unterschiedenen Funktion jetzt in der Auflösung des Perzipierten in Perzeption, in der Vermischung von Inhalt und Akt der Wahrnehmung, in der Nichtunterscheidung von objektiver und subjektiver Existenz sieht (vgl. Anm. 76, 90, 156, 302, 308). Iie neue Fassung del Anmerkungen

1; Tun mir grsperrt.

Kantetadiou XIV. 
156, 191, 282 belehrt uns nun darüber, wie Lipps za seiner Anffassung der Entstehung von Humes Irrtum gekommen ist: dieser verfallt jener Verwechselung, weil er nicht zur Erkenntnis des Objektivitätsbowusstseins als des "mit jedem sonstigen Datum unseres Bewusstseins Unvergleichbaren " vorgedrungen, sondern über die Reduktion der Objekte auf subjektive Wahrnehmungen nicht hinausgekommen ist. Er hat das "von jedem blossen Perzipieren, oder Haben von Bewusstseinsinhalten toto coelo verschieden ${ }^{1}$ ) Wesen des Denkens, oder, was dasselbe sagt, ${ }^{1}$ ) er" hat "den Begriff des dem Inhalte transscendenten „Gegenstandes" "1) nicht gefunden.

Die Frage, ob Hume die Realität des Aussendings anerkannt oder bezweifelt habe, bildet nun einen Streitfall zwischen Lipps, dessen jetzige Auffassung der letzteren Annahme zuneigt, und Hönigswald (a. a. 0.), der die Anschauung vertritt, dass Hume an den die Dinge der Erfahrung begründenden Aussendingen festgehalten habe. Da ich dieses Problem der Humeinterpretation, zu dem jetzt auch Cassirer a. a. O. (bes. S. 676 [Anm. 33]) das Wort ergreift, gelegentlich einer Anzeige von Hönigswalds Schrift in den Kantstudien näher zu erörtern gedenke, so sehe ich hier von einer Stellungnahme zu dieser speziellen Frage der Auslegung ab. Von Interesse ist zunächst nur, dass Lipps mit der zuletzt aus Anmerkung 282 angeführten Kritik an Humes Perzeptionstheorie eine Auffassung von dem Verhältnis zwischen Wahrnehmung, Denken und Gegenstand bekundet, die nicht als eine gradlinige Fortsetzung seines Weges von Hume hinweg nacb Kant hin betrachtet werden kann.

Während die Kritik des Humeschen Psychologismus, welche die Unabhängigkeit des Logischen, des Denkens in Gesetzen von blosser Wahrnehmung fordert, gradeswegs der Einheitlichkeit der Auffassung zustrebt, welche allein die Lösung des Erkenntnisproblems gewährleisten kann, bringt der weiteren Verfolg der Betrachtung, wobei Lipps das "Haben von Bewusstseinsinhalten" und das "Denken“ als zwei wesentlich verschiedene Fähigkeiten trennt, uns vor einen Dualismus, der die Lösung - wenigstens hinausschiebt. Sie wird nicht zu finden sein, solange man bei dieser psychologischen 'Trennung der Erkenntnisvermögen stehen bleibt. Nicht darum handelt es sich, ob "unsere gewöhn-

1) Von mir gesperrt. 
lichsten "Einbildungen “ in letzten Grunde denselben Faktoren ihr Dasein verdanken, wie unsere sichersten Erfahrungserkenntnisse“ (2. Aufl., Anm. 191) - diese genetisch interessierte Frage kann nur eine biologisch-metaphysische Scheinlösung finden - sondern die Frage muss, wenn anders eine „verstandesmässige“, kritische Lösung überhaupt erwartet werden soll, so gestellt werden: wie ist die als unbestreitbares Faktum in den Wissenschaften, vorab in der Mathematik und der reinen Naturwissenschaft vorliegende, soweit Erfabrung gewordene Erkenntnis (deren letzte „Wurzeln“ nur biologisch aufgegeben, erkenntnismässig aber unerreichbar sind) möglich? Das heisst: wie ergeben sich ihre Bedingungen in einheitlichem, notwendigem Zusammenhang, ohne dass die zur Eiffassung der ursprünglichen .Synthesis praktisch erforderliche Analyse über- oder unterlogische Faktoren, überhaupt etwas anderes als die logische Struktur der vorliegenden Erkenntnis ins Auge fasst? Diese letztere Forderung ist, das muss betout werden, eine unerlässliche, weil Frkenntnis nur logisch geformt werden kann. Anstatt des subjektiven „Vermögens“ wird die sachliche Möglichkeit "zu suchen, statt nach dem "Denkakte“ nach der Funktion der den Gegenstand der Erfahrung überhaupt konstituierenden Voraussetzungen zu fragen sein.

Es ist schon viel gewonnen, wenn das Denken in seiner Unabhängigkeit von den Daten der sinnlichen Erfahrung erkannt wird. Soll jedoch die Erkenntnislehre hier nicht in zwei Gebiete zerfallen, von denen das eine, weil unterlogisch („blosses Haben von Bewusstseinsinhalten “), jeder Erfassung durch die Erkenntnis überhaupt entzogen wäre, so muss die notwendige Folgerung aus der einmal anerkannten „Übcrlegenheit“ des gesetzmässigen Denkens gezogen und dies als Bedingung aller und jeder Erfahrung eingesetzt werden. Es bildet somit anch die Grundlage für ein jedes „Haben von Bewusstseinsinhalten“ und „Datum unseres Bewusstseins", indem diese, sofern sie überhaupt für unser Bewusstsein in betracht kommen sollen, Ohjekte sind; wobei die Frage, ob und inwiewcit sie in dem individuellen Subjekt als solche erzeugt werden, erst in zweiter Linie steht und cine solche der Psychologie ist. Das reine Denken, das Lipps mit Recht als das Jenken vou Gegenständen bezeschnet, wird seinem IVesen nach erst dann richtig erfasst werden, wenn es aufhört, ein gewissermassen sublimierter 'Teil unser's Frkenntnisvermögens zu sein, sondern wenn es als die Bedingung der Möglichkeit einer jeden 
Erfabrung auftritt. Hierbei ist freilich eines festzuhalten: es kann objektiv nicht halbe und ganze, embryonale und ausgewachsene "Erfahrung" geben; "Erfahrung" kann nicht in Teile von verschiedener Gewissheit zerfallen: sie ist alsdann eben keine "Erfahrung ${ }^{*}$, sondern subjektiver und somit durchaus unkontrollierbarer Zustand. Als "Erfahrung“ darf nur Erkenntnis gelten, sofern sie Wissensc haft geworlen, und sei es solche von den „einfachsten“ Dingen. Solald dieser Massstab zugelassen ist, scheidet sich klar das Arbeitsgebiet der Erkenntnistheorie, als der wahren "Lehre vom Verstande“, und das der Metaphysik. In das letztere fällt alles, was nicht als klar unrissener, synthetisch erzeugter Gegenstand der Erfahrung, d. i. Erkenntnis, angesprochen werden kann. Dann wird der Zwiespalt zwischen einer "niederen " und einer "höheren" Art der Erkenntnis verschwinden und nur noch Erkenntnis schlechthin in betracht kommen. Sobald das reine Denken als das Grundgefüge der Erfahrung, als die Bedingung der Möglichkeit des Erfahrungsgegenstandes überhaupt erfasst ist, scheidet einerseits jegliches "untere“ oder Unbewusstsein, als günzlich ausserhalb jeder möglichen Erfahrung liegend, aus, andererseits wird jeder jenseits oder über dem Erfahrungsgegenstand liegende „transscendente Gegenstand“ als bloss negativer Begriff gelten müssen, dem in unserer Erkenntnis kein „Datum“ entspricht. Denn ich kann die Tatsache, dass Lipps (2. Auflage, Anm. 282) das Wesen des Denkens mit dem „Begriff des dem Inhalte transscendenten $\left.{ }^{1}\right)$ Gegenstandes“ gleichsetzt, nur dadurch erklären, dass er das reine Denken nicht als die Grundlage der Erfahrung überhaupt, d. i. als Bedingnng der Möglichkeit der Gegenstände unserer Erkenntnis ansprach, sondern es als eine Art höheres Vermögen von dem „blossen Perzipieren" unterschied; als ein Vermögen, dem auch eine höhere Aufgabe als ein „bloss“ erfahrungsmässiges Erfassen von „Bewusstseinsinhalten" - dem der "Gegenstand selbst" zugewiesen werden müsse. Damit, wenn ich mit dieser Auslegung Lipps' Meinung getroffen habe, ist jedoch der. Schritt vom reinen zum absoluten Denken, vom Gegeustand der Erfahrung. zu dem der Metaphysik gemacht. Denn es ist nicht zu ersehen, welche Brücke von unserer Erkenntnis zum „transscendenten Gegenstande“, der nur als das aufgegebené $x$ der Erkenntnisgleichung Sinn hat,

1) Von mir gesperrt. 
hinführen soll. Die Eindeutigkeit, Geschlossenheit, besser: die systematische Einheit der Lösung, bei der kein Teil abseits gestellt werden muss; diese Einheit, diese Notwendigkeit der Lösung des Erkenntnisproblems, das alleinige Kriterium der Lösung, ist nicht erreicht. Lipps entzieht das Denken dem unsicheren Boden der subjektiven Wahrnehmung, worauf Hume es zu gründen versucht hatte: doch scheint er noch in der alten psychologischen Problemlage zu sehr befangen, um ihm nun sogleich seine wahre Stellung als Bedingung der Erkenntnis anzuweisen: es bleibt, als ob er ster zwar, ein Teil des subjektiven Erkenntnisvermögens, und indem „die apriorische Gesetzmässigkeit des Denkens, die Eines ist mit der Gesetzmässigkeit der gedachten Gegenstände“, nicht zweifellos als die Beding ung der Möglichkeit der letzteren gesetzt wird, muss sie als von der des "transscendenten Gegenstandes" abhängig gedacht erscheinen.

Ist nun auch damit die Lösung im Sinne Kants hinausgeschoben, so bleibt doch die Gewähr, dass Lipps auf sie losgeht. Den entscheidenden Schritt: die Anerkennung der Unabhängigkeit des Denkens von jeglicher "Perzeption" hat er getan. Und dass er weitere tun wird, schliesse ich aus der Formulierung eben derjenigen Stellen, deren Rationalismus ich angreifen musste. Ich bin der Hoffnung, dass die Abwendung vom transscendenten Gegenstande zum transscendental begründeten nur noch eine Frage der Zeit sein wird.

Die sonst noch von Lipps in der 2. Auflage in den Anmerkungen vorgenommenen Änderungen sind weniger wichtig als die oben hervorgehobenen, auch sind ihrer nur noch wenige zu verzeichnen. Gewöhnlich enthalten sie eine Rechtfertigung für die in der Neubearbeitung auftretenden Änderungen der Interpretation. Diese stellen, soweit meine Stichproben reichen, ${ }^{1}$ ) durchaus einen Fortschritt dar. Die Kontrolle ist an zweifelhaften Stellen überall ermöglicht; wie schon in der 1. Auflage, durch Anführung des Originaltextes und Besprechung der Übersetzung sowohl als des sachlichen Zusammenhanges mit den Humeschen Leitgedanken, oder dadurch, dass die Lippsschen Zusätze in eckige Klammern gesetzt werden. Die Anmerkungen sind in der neuen Fassung

2) Ich benutzte die Ausgabe von Green-Grose von 1898, während Lipps die von $1878 \mathrm{zu}$ Grunde legte; doch glaube ich, dass die erstere keine nennenswerten Änderangen gegenüber der letzteren aufweist. 
der Gedanken in noch höherem Grade von grossem propädeutischem Wert als in der alten; die Zusätze schaffen glücklich gedankliche Übergänge füir den Leser. Im allgemeinen war jedoch für den Text der Übersetzung nur an wenigen Stellen eine sachliche, meist eine bloss stilistische Revision nötig; im ganzen liegt derselbe Übersotzungstext vor. Indes giebt die Arbeit, die Lipps an dem neuen Buche geleistet, ihm das Recht, den Titelaufdruck der 1. Auflage: „... übersetzt von E. Köttgen, die Übersetzung überarbeitet von Th. Lipps", umzuändern wic oben ersichtlich und die Bemerkung der Vorrede über das Verhältnis seiner Überarbeitung zur ursprünglichen Übersetzung E. Köttgens zu streichen. - Im übrigeu ist er bemüht geweseu, die neue Ausgabe so zu gestalten, dass sie in der äusseren Auordnung möglichst wenig von der alten abweicht: Die in den Anmerkungen nötig gewordenen Änderungen, bes. auch Streichungen, sind so ausgeglichen, dass Zahl und Nummer der Anmerkungen in allgemeinen in der neuen Auflage sich gleich geblieben sind, wodurch auch die Verweise im Register am Ende vor Verschiebungen bewahrt wurden, desgleichen auch mit verhältnismässig wenigen Abweichungen die Seitenanordnuug, sodass das neue Buch ganz dazu angetan ist, unmerklich an die Stelle des alteu rücken zu können.

An dem trefflichen Werke, das auch für den des Englischen kundigen Humeleser unentbehrlich sein wird, bleibt nur IVeniges auszusetzen. Das bei Green-Grose nach den Originalausgaben kursiv Gedruckte sähe man gerne auch bei Lipps überall entsprechend markiert; die Änderungen der Neuauflage scheinen im "Namen- und Sachregister" (S. 365-380) nicht durchweg berücksichtigt; ich vermisse z. B. unter Perzeption einen Hinweis auf die neue Anmerkung 176 und die geänderte $302 \mathrm{zl}$ „internal perception"; ferner finde ich interessante Begriffe wie "survey" oder "view" (hierzu die nete Anmerkung 219, welche die neve Übersetzung: = "Anffassungsakt" gegenüber der alten $=$ „Bild" rechtfertigt) nicht in Register vertreten. Die iuzwischeu erschienene Litteratur scheint nicht verwertet worden zu sein; wenigstens äussert sich Lipps unter dem 'lexte nicht. zu dem, was z. B. Quast, Der Begriff des belief bei David Hume (1903)') in seinen Anmerkungen, so etwa S. 23, Anm. 1,2) S. 52, Anm. 3

1) Siehe meine Anzeige im Literarischen Zentralblatt 1906, No. 49, Sp. $1663 \mathrm{f}$.

2) Quast zitiert hier falsch ,jeden Glauben" statt ,jedes Glauben". 
über einzelne Stellen des Textes der 1. Auflage bemerkt. Doch sind das unwesentliche Ausstellungen, die bei einem Neudruck leicht berücksichtigt werden können.

Der zweite Band bringt uns die Lebensphilosophie Humes: mit diesem Wort ist, glaube ich, am besten die Gesamtheit dieser seiner Ausführungen über alle möglichen Fragen der Ethik und Rechtsphilosophie auf psychologischer Grundlage bezeichnet. Die von Hume selbst vorgenommene Zweiteilung ist nicht streng $\mathrm{zu}$ verfolgen; die Gebiete greifen häufig ineinander über: der „miscellaneous way of reasoning", ein Hauptreiz geistreicher Konversation, für Humes Art der Darstellung auch im erkenntnistheoretischen Teil des Treatise schon bezeichnend, kommt bei dem zweiten und dritten Teil, dessen Gegenstände der litterarischen Formung sich ja auch williger darbieten, voll zur Auswirkung. Sie sind für das Verständnis der späteren inhaltlich verwandten, überhaupt der nicht erkenntnistheoretischen Schriften Humes, die in erster Linie seinen litterarischen Ruhm begründeten, nicht minder wichtig, als der erste Teil es für die sogleich erfolgreiche und folgenreiche Inquiry concerning human understanding ist. Lipps hat mit der Veröffentlichung des 2. Bandes sein 1895 begonnenes Unternehmen in dankenswerter Weise zum Abschluss gebracht.

Die Grundsätze, nach denen Lipps bei der Übersetzung verfuhr, sind die aus der Vorrede zum ersten Bande bekannten. Es gewährt einen ästhetischen Genuss, den Urtext mit seiner dentschen Übertragung zu vergleichen. Ich zweifle nicht daran, dass jene Worte der Vorrede zur 1. Auflage von Bd. I, die in der 2. Auflage weggelassen sind, jetzt für den 2. Band Geltung baben: „Was das Verhältnis der „Überarbeitung“ zur Übersetzung angeht, so bemerke ich, dass die Rücksichtslosigkeit der ersteren die Urheberin der letzteren von der Verantwortung für das schliessliche Ergebnis befreit." Die Bearbeitung steht auf dem Standpunkt, dass nur durch freie Umformung und Auflösung des euglischen Satzgefuiges, durch exakte Übertragung der Worte der gedanklichc Kern von Hunıes Perioden rein wiedergegeben werden kann. Die Übersetzung bedentet eine gedankliche Neuschöpfung, ohne doch in geringsten über den Rahmen des Ursinnes hinauszugehen, ohne Humes Begriffen eine nicht in ihrer Grundform liegeude sluance zu geben. Ich habe nach Anglizismen gespürt 
und lei vielen Stichproben uicht den leisesten Anklang daran gefunden. Die Kontrolle ist wie bei Band I ermöglicht.

Nur ein Missverständnis, eine im Englischen nicht ungewöhuliche syntaktische Erscheinung betreffend, ist zu notieren: Lipps sagt Anmerkung 78, S. 293: „Im Text heisst es: ingraft them on those other duties. Der Sinn verlangt aber die Umkehrung: ingraft on them those other duties. - Solche sinnstörende Schreib- oder Druckfehler sind auch sonst in der Ausgabe von Green und Grose stehen geblieben." Ich glaube, die 4 Nenausgaben, die seit der von 1878, die Lipps benutzte, erschienen sind, haben den Text von wirklichen Fehlern im wesentlichen gereinigt; wenigstens finde ich in meinen Exemplar der letzten von 1898 keine mehr: dagegen steht auch im neuesten Abdruck noch: to ingraft them on those other duties; das ist aber kein "sinnstörender Fehler": wir haben es vielmehr mit einem syntaktisch regelrechten Satz zu tun, zu dem der von Lipps als richtiger vorgeschlagene nur eine eben zulässige Variante bildet. Beide können übersetzt werden: „... auf sie [scil.: die Naturgesetze] jene anderen Pflichten ... aufzupfropfen " [Lipps a. a. 0.]. Das Missrerständnis Lipps', die Präposition on könne dem Pronomen, auf das sie sich bezieht, nicht nachstehen, ohne von dem Leser notwendig den Worten those other duties beigeordnet $\mathrm{zu}$ werden, wodurch der ganze Sinn zur Unverständlichkeit verschoben werden müsse, kommt durch die Erwägung in Wegfall, dass die englische Satzbetonung nach to ingraft them on eine "Cäsur" fordert, sodass für den Hörer und für denjenigen Leser, der beim Lesen in Gedanken die Sätze gesprochen hört, die „Prä“position ohne weiteres als dem voraufgehenden Pronomen beigeordnet erscheint (Postposition). Diese Stellung der logisch zum Verb gehörigen "Prä"position hinter dem von ihr abhängigen Nomen ist nämlich eine bekannte syntaktische Erscheinung, zu der die vom Verb losgelöste, dem Nomen voranstehende Präposition, wie sie Lipps in seiner Änderung giebt, bloss eine Variante bildet. Der Urtext lautet, genau übersetzt: „ihnen jene anderen Pflichten ... aufzupfropfen"; die Änderung von Lipps jedoch: "auf sie jene anderen Pfichten . . . zu propfen". Das kommt jedoch sachlich auf dasselbe hinaus.

Nicht der Text des 2. Bandes, wohl aber die Anmerkungen haben es entgelten müssen, dass Lipps' Teilnahme für Hume nicht mehr so stark ist wie zur Zeit der Bearbeitung der 
1. Auflage von Band I. Man vergleiche die Zahl der Aumerkungen: im 1. Band: 346 z. T. sehr ausführliche, bisweilen fast kleine Abhandlungen darstellende Fussnoten: im 2. (stärkeren) Band: 92 zum grössten Teil sehr knappe Randbemerkungen. Das mag teilweise im Gegenstand des Textes seinen Grund haben: Humes Erkenntnistheorie ist weitaus interessanter 'und geschichtlich bedeutsamer, als der Inhalt des 2. und 3. Buches (Of the passions, Of morals). Aber doch glaube ich: wenn 1904 nicht schon von Book I die Bearbeitung in 1. Auflage mit jenem programmatischen Vorwort und den von reger Teilnahme an dem sachlichen Kern zeugenden zahlreichen Anmerkungen vorgelegen hätte, so würde Lipps viel seltener das Wort ergriffen haben, als es in der 2. Auflage geschehen. Ich komme zu dieser Vernutung ausser durch die Beobachtung der oben besprochenen Änderungen an der 1. Auflage noch durch die Wahrnehmung, dass die Anmerkungen $35,51,58,76,82,88$ des 2 . Bandes Wendungen enthalten, die auch an der formalen Struktur von Humes Ausführungen oft recht scharfe Kritik üben, während das Vorwort zur 1. Auflage von Band I aussagte, „dass Hume zu den klarsten Schriftstellern gehört". Man muss daran denken, dass - Kant bei aller Kritik der Sache wenigstens immer noch den „unnachahmlich schönen Vortrag" Humes rühmte. Sic transit gloria . . . Alles in allem: wir haben keine Ursache, darüber zu murren, dass Lipps in der 1. Auflage von Band I für Hume so eifrig eintrat. Wir verdanken dieser warmen Teilnahme den trefflichen ersten Band mit seinen zahlreichen Anmerkungen und - seine zweite Auflage, die nicht nur ein durchgesehenes, sondern auch ein auf ungemein lehrreiche Art revidiertes Buch ist; ferner den zweiten Band, der dem Gefühl entsprang, dass einmal Begonnenes jedenfalls unter Dach gebracht werden müsse. Wenn eine objektive Würdigung Humes in Zukunft immer weiter um sich greift, so wird Lipps sein redlich Teil dazu beigesteuert haben. 